\title{
Combinatorial Obstructions to the Lifting of Weaving Diagrams
}

\author{
Jürgen Richter-Gebert \\ Auf dem Wingert 3, D-64380 Roßdorf, \\ Federal Republic of Germany
}

\begin{abstract}
This paper deals with various connections of oriented matroids [3] and weaving diagrams of lines in space [9], [16], [27]. We encode the liftability problem of a particular weaving diagram $\mathscr{D}$ on $n$ lines by the realizability problem of a partial oriented matroid $\chi_{\mathscr{D}}$ with $2 n$ elements in rank 4 . We prove that the occurrence of a certain substructure in $\mathscr{D}$ implies that $\chi_{\mathscr{D}}$ is noneuclidean in the sense of Edmonds, Fukuda, and Mandel [12], [14]. Using this criterion we construct an infinite class of minor-minimal noneuclidean oriented matroids in rank 4. Finally, we give an easy algebraic proof for the nonliftability of the alternating weaving diagram on a bipartite grid of $4 \times 4$ lines [16].
\end{abstract}

\section{Introduction}

This paper connects two different topics on the borderline of geometry and combinatorics: oriented matroids and weaving diagrams. Oriented matroids, on the one hand, are combinatorial abstractions of dependencies in linear vector spaces. They form a broad setting to deal with the combinatorial properties of various types of configurations, such as arrangements of (pseudo)hyperplanes, configurations of points, linear programs, or convex polytopes. Many properties of these kinds of configurations can be derived by inspection in the underlying oriented matroid. For instance, the face lattice of a convex polyhedron or the optimal solution of a linear programming problem can be computed this way. For an extensive and readable introduction to the theory of oriented matroids the reader is referred to [3].

Weaving diagrams, on the other hand, form a description of the combinatorial properties of arrangements of lines in 3-space. If we consider an arrangement of lines $\mathscr{L}^{\prime}=\left\{l_{1}^{\prime}, l_{2}^{\prime}, \ldots, l_{n}^{\prime}\right\}$ in 3-space and project it onto a plane $P$ by a projection $\pi$, then we usually (if no line $l_{i}^{\prime}$ passes the center of the projection) get a line 
arrangement $\mathscr{L}:=\pi\left(\mathscr{L}^{\prime}\right)=\left\{\pi\left(l_{1}^{\prime}\right), \pi\left(l_{2}^{\prime}\right), \ldots, \pi\left(l_{n}^{\prime}\right)\right\}$ in the plane. Moreover, the projection induces an above-below relation $\Delta$ for each pair $\left(\pi\left(l_{i}^{\prime}\right), \pi\left(l_{j}^{\prime}\right)\right)$ of lines intersecting in the plane $P$. The pair $(\mathscr{L}, \Delta)$ is usually called a planar layout [9] or a line weaving [27]. The line weaving $(\mathscr{L}, \Delta)$ forms a kind of sketch of our original line arrangement $\mathscr{L}^{\prime}$. If we replace $\mathscr{L}$ in $(\mathscr{L}, \Delta)$ by the equivalence class $[\mathscr{L}]$ of all planar (pseudo)line arrangements which are combinatorially equivalent to $\mathscr{L}$, we obtain an even more abstract picture of our original arrangement: the weaving diagram $([\mathscr{L}], \Delta)$ (see [9] and [16]).

It is a well-known fact that the combinatorial types of pseudoline arrangements (affine or projective) are well described in terms of rank 3 oriented matroids (actually this is just a special case of the "Folkman and Lawrence Representation Theorem" [11]). Therefore we can represent $[\mathscr{L}]$ by a suitable oriented matroid $\chi[\mathscr{L}]$.

Using this fact we give an intrinsic definition of weaving diagrams. Roughly speaking, a weaving diagram $\mathscr{W}$ is a pair $(\chi, \Delta)$, where $\chi$ is a rank 3 oriented matroid describing a pseudoline arrangement and $\Delta$ is an "above-below" choice for each intersection of two (pseudo)lines in the plane. A weaving diagram $(\chi, \Delta)$ is called liftable if a spatial arrangement of lines $\mathscr{L}^{\prime}:=\left\{l_{1}^{\prime}, l_{2}^{\prime}, \ldots, l_{n}^{\prime}\right\}$ and a projection $\pi$ such that $\pi\left(\mathscr{L}^{\prime}\right)$ is combinatorially equivalent to $\chi$ and the abovebelow relations induced by the projection $\pi$ correspond to those of $\Delta$ exists. Figure 1(a) shows a liftable weaving diagram, while Fig. 1(b) shows a nonliftable one.

Although, during our process of replacing an essentially three-dimensional object by a combinatoric pattern $\mathscr{W}=(\chi, \Delta)$ of dimension 2 , a lot of information seems to be lost, $\mathscr{W}$ still contains much information about the original line arrangement. For instance, if $\mathscr{L}^{\prime}$ was originally a line arrangement in projective 3-space the complete class of its flexible isotopic line arrangements can be described in terms of the underlying weaving diagrams. Recent studies by Crapo and Penne [9], [17], Viro [25], [26], and Mazurovskii [15] relate the movements of the lines in space to some kind of "Reidemeister-like" moves on the weaving diagrams considered as topological links. However, while performing such moves weaving diagrams that are no longer liftable can occur. This is one reason why it is important to study the liftability problem of weaving diagrams. It is still a main question whether for every pair of line arrangements that admits a flexible isotopy there also exists a rigid isotopy (leaving the lines straight). Here the liftability of

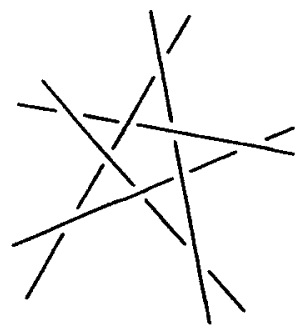

(a)

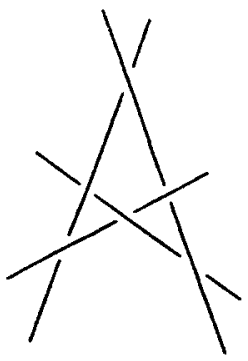

(b)

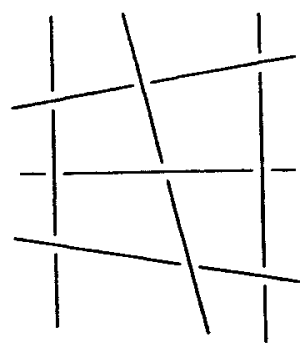

(c)

Fig. 1. Liftable and nonliftable weaving diagrams. 
the underlying weaving diagrams forms an important constraint for the rigid isotopies (see [9]).

Another motivation for studying the liftability question arises from considerations of structural rigidity. If a given weaving of proper lines $(\mathscr{L}, \Delta)$ is polarized a situation occurs where the original lines of $\mathscr{L}$ correspond to points $\mathscr{P}$ in the plane. The above-below relations on the lines translate to a tensegrity framework, where the lines correspond to the joins of the framework and the "above/below choices" translate to cables and struts between the joins, depending on the choosen polarity. The nonliftable line weavings correspond to those bar and joint frameworks that admit a proper self-stress [27]. There are frameworks which are rigid if the points are in generic position but admit an infinitesimal motion for certain special positions of the points. This translates to the fact that there are line weavings that are liftable if the lines are in generic position but become nonliftable in a special position of the lines. The partial weaving diagram given in Fig. 1(c) is nonliftable if and only if the six lines are tangent to a planar conic section.

Liftability has also been studied in connection to spatial interpretations of two-dimensional projections of polytopal figures [10], [13], [24]. There the problem was mainly attacked by studying the underlying algebraic structure [10] or the underlying (unoriented) matroid structure [13], [24].

In this paper we translate the liftability problem for a weaving diagram into the realizability problem of a certain partial oriented matroid. We associate to each weaving diagram $\mathscr{W}$ on $n$ lines a partial rank 4 oriented matroid $\chi_{\mathscr{W}}$ on $2 n$ elements which is realizable if and only if $\mathscr{W}$ is liftable. This connection turns out to be fruitful for both oriented matroid theory and the theory of weaving diagrams. On the one hand, some well-understood methods in oriented matroid theory for attacking the realizability question can be used to decide the liftability of weaving diagrams. On the other hand, even relatively easy constructions of nonliftable weaving diagrams provide interesting classes of examples of nonrealizable oriented matroids in rank 4. Moreover, the weaving diagrams give an easy way of visualizing the geometric content of these examples.

As an application of such constructions we present (Section 3) an infinite minor-minimal class of noneuclidean oriented matroids in rank 4 . Noneuclidean oriented matroids arise in the context of the linear programming equivalent for oriented matroids as given by Bland [4]. An oriented matroid program is noneuclidean in the sense of Edmonds, Fukuda, and Mandel [12], [14] if it admits an infinite nondegenerate cycling of increasing pivot operations. We construct a certain class of nonliftable weaving diagrams where a kind of geometric cycling on the line diagram $\mathscr{W}$ translates to noneuclideanness of the partial oriented matroid $\chi_{\mathscr{W}}$. By giving an infinite minor-minimal class of such examples we construct an infinite class of noneuclidean partial oriented matroids in rank 4 such that every minor is euclidean. To drop the adjective "partial" we apply a construction (given in Section 2) of how we can obtain a unique oriented matroid from a given (proper) line weaving. Our results for oriented matroids contrast with the case of ordinary matroids where it was proved by Bachem and Wanka that for rank 4 matroids noneuclideanness can be characterized by excluding a unique minor on eight elements [1]. 
In Section 4 we exemplify how nonrealizability proofs for oriented matroids can be applied to prove nonliftability for weaving diagrams. We give an easy algebraic proof for the nonliftability of the alternating weaving diagram on a bipartite grid of four vertical and four horizontal lines. An alternative proof of this fact has been given by Pach et al. [16]. In contrast to this proof, which involves many special considerations and case distinctions which are very geometric in nature, the proof presented here is based on some simple determinant identities on a matrix that coordinatizes the liftable situation.

\section{Weaving Diagrams and Oriented Matroids}

In this section we explore various connections of (partial) oriented matroids and weaving diagrams. Oriented matroids of rank 3 are essentially equivalent to arrangements of pseudolines in the projective plane. Therefore, from a combinatorial point of view, it makes sense to use oriented matroids as a description of the underlying combinatoric structure of the planar line arrangement belonging to a weaving diagram.

Let $X$ be a collection of vectors $X:=\left(x_{1}, x_{2}, \ldots, x_{n}\right) \in\left(\mathbb{R}^{d}\right)^{n}$ and $E:=\{1, \ldots, n\}$. The mapping

$$
\begin{aligned}
\chi_{X}: E^{d} & \rightarrow\{-1,0,+1\}, \\
\left(\lambda_{1}, \ldots, \lambda_{d}\right) & \mapsto \operatorname{sign} \operatorname{det}\left(x_{\lambda_{1}}, \ldots, x_{\lambda_{d}}\right)_{X}
\end{aligned}
$$

is called the (realizable) oriented matroid of $X$. In general, oriented matroids are combinatorial structures that form a generalized setting of linear dependencies in vector configurations.

For the general definition of oriented matroids it is useful to make calculations on the fuzzy ring $G F_{3}:=(\{-1,0,+1, *\}, ;+)$ as described in [8]. Here the commutative operations " + " and "." simulate the behavior of the signs of real numbers under addition and multiplication. The symbol "*" reflects the situation when a certain sign is not known. Addition and multiplication in $G F_{3}$ are given in the following two tables:

\begin{tabular}{|r|cccc|}
\hline \multicolumn{1}{|c|}{} & 0 & +1 & -1 & $*$ \\
\hline 0 & 0 & 0 & 0 & 0 \\
+1 & 0 & +1 & -1 & $*$ \\
-1 & 0 & -1 & +1 & $*$ \\
$*$ & 0 & $*$ & $*$ & $*$ \\
\hline
\end{tabular}

\begin{tabular}{|r|cccc|}
\hline+ & 0 & +1 & -1 & $*$ \\
\hline 0 & 0 & +1 & -1 & $*$ \\
+1 & +1 & +1 & $*$ & $*$ \\
-1 & -1 & $*$ & -1 & $*$ \\
$*$ & $*$ & $*$ & $*$ & $*$ \\
\hline
\end{tabular}

Now an oriented matroid can be defined by: 
Definition 1.1. For a finite set $E$ an alternating map $\chi: E^{d} \rightarrow\{-1,0,+1, *\}$ is called a partial oriented matroid on $E$ if

$$
\sum_{i=1}^{d+1}(-1)^{i} \cdot \chi\left(\lambda_{1}, \ldots, \lambda_{i-1}, \lambda_{i+1}, \ldots, \lambda_{d+1}\right) \cdot \chi\left(\lambda_{i}, \tau_{1}, \ldots, \tau_{d-1}\right) \in\{0, *\}
$$

in the fuzzy ring $(\{-1,0,+1, *\}, \cdot,+)$ for any $\lambda \in E^{d+1}$ and $\tau \in E^{d-1}$. If $\operatorname{Im}(\chi) \subseteq$ $\{-1,0,1\}$ the map $\chi$ is called an oriented matroid. If $\operatorname{Im}(\chi) \subseteq\{-1,1\}$ the map $\chi$ is called a uniform oriented matroid. $d$ is called the rank of the oriented matroid.

A partial oriented matroid $\chi$ is called realizable if there is an $X \in\left(\mathbb{R}^{d}\right)^{n}$ with $\chi_{x}(\lambda)=\chi(\lambda)$ for every $\lambda \in \chi^{-1}(\{-1,0,+1\})$. An oriented matroid is called acyclic if there exists a $\lambda \in E^{d-1}$ such that $\chi(\lambda, E) \subseteq\{0,+1\}$. Later we translate the liftability of a weaving diagram $\mathscr{W}$ into the realizability of a certain partial oriented matroid $\chi_{\mathscr{W}}$.

We now explain how to encode the combinatorial type of an affine weaving diagram in terms of oriented matroids. Each line $l$ in the affine plane $\mathbb{R}^{2}$ not containing the origin can be uniquely described by the equation $A x+B y+1=0$, where $A$ and $B$ are suitable chosen parameters. The open half-space of $\mathbb{R}^{2} \backslash$ containing the origin is called the positive side of $l$. We can think of the line $l$ as being directed by the rule that while passing along $l$ in a positive direction the positive side of $l$ is always on the left. If $\mathscr{L}:=\left(l_{1}, l_{2}, \ldots, l_{n}\right)$ is an affine arrangement of lines $l_{i}:=\left\{(x, y) \mid A_{i} x+B_{i} y+1=0\right\}$ in the plane, then the oriented matroid $\chi_{X(\mathscr{L})}$ of

$$
X(\mathscr{L}):=\left(\begin{array}{ccc}
A_{1} & B_{1} & 1 \\
A_{2} & B_{2} & 1 \\
\vdots & \vdots & \vdots \\
A_{n} & B_{n} & 1 \\
0 & 0 & 1
\end{array}\right)
$$

encodes the combinatorial structure of the affine line arrangement $\mathscr{L}$. Two line arrangements $\mathscr{L}_{1}$ and $\mathscr{L}_{2}$ generate the same oriented matroid $\chi_{x\left(\mathscr{L}_{1}\right)}=\chi_{x\left(\mathscr{L}_{2}\right)}$ if and only if following any line $l_{i}$ in a positive direction the remaining lines occur in the same order for both arrangements $\mathscr{L}_{1}$ and $\mathscr{L}_{2}$. In the above matrix $X(\mathscr{L})$ each row represents homogeneous coordinates of one of the (oriented) lines. The last row $(0,0,1)$ encodes the position of the line at infinity of the affine plane $\mathbb{R}^{2}$. Notice that $\chi_{X(\mathscr{L})}$ is acyclic, since the origin lies on the same side of all lines. In what follows we assume that all line arrangements $\mathscr{L}$ are simple, i.e., at most two lines meet in a point and no two lines are parallel (in other words the oriented matroid $\chi_{X(\mathscr{L})}$ is uniform). We define $\bar{E}:=\{(i, i) \mid i \in E\}$. To encode a weaving diagram we now add an alternating mapping $\Delta: E^{2} \backslash \bar{E} \rightarrow\{-1,0,+1, *\}$ to the structure, telling whether at each point of intersection of two lines $(i, j) \in E^{2} \backslash \bar{E}$ the line $l_{i}$ lies over $l_{j}$ (i.e., $\Delta(i, j)=+1$ ), line $l_{i}$ lies under $l_{j}$ (i.e., $\Delta(i, j)=-1$ ), line $l_{i}$ and $l_{j}$ coincide (i.e., $\Delta(i, j)=0$ ), or if we do not care about the relation of $l_{i}$ and $l_{j}$ (i.e., $\Delta(i, j)=*$ ). 
More combinatorially we define:

Definition 1.2. An affine weaving diagram $\mathscr{W}:=(\chi, \Delta)$ on $E:=\{1, \ldots, n\}$ consists of a uniform acyclic rank 3 oriented matroid $\chi$ on $E \cup\{n+1\}$ and an alternating mapping $\Delta: E^{2} \backslash \bar{E} \rightarrow\{-1,0,+1, *\} . \chi$ is called the affine arrangement of $\mathscr{W}$ and $\Delta$ is called the weaving pattern of $\mathscr{W} . \mathscr{W}$ is called complete if $\operatorname{Im}(\Delta) \subseteq\{-1,0,+1\}$. $\mathscr{W}$ is called uniform if $\operatorname{Im}(\Delta) \subseteq\{-1,+1\}$.

By the above considerations it is straightforward how to encode the information contained in pictures like the ones of Fig. 1 in a corresponding affine weaving diagram $\mathscr{W}:=(\chi, \Delta)$. We arbitrarily choose a coordinate system with the origin not on the lines, compute the corresponding oriented matroid $\chi$, and define the weaving pattern $\Delta$ consistently to the picture.

We now express the liftability of an affine weaving diagram $\mathscr{W}$ by the realizability of an associated partial rank 4 oriented matroid $\chi_{\mathscr{W}}$. Therefore we first discuss how the line diagram $\mathscr{L} \subset \mathbb{R}^{2}$ can be considered as arising from a spatial line arrangement $\mathscr{L}^{\prime}$ in $\mathbb{R}^{3}$ by orthogonal projection onto the $(x, y)$-plane $\mathbb{R}^{2}$. To avoid the situation that a line in $\mathscr{L}^{\prime}$ projects down to a line containing the origin, we assume that all lines to be considered do not hit the $z$-axis $l_{z}:=\{(x, y, z) \mid x=y=0\}$. We also assume that none of the lines in $\mathscr{L}$ is parallel to $l_{z}$, to avoid the occurrence of lines which project to a single point. By $\pi$ we denote the orthogonal projection $\pi(x, y, z)=(x, y)$ on the $(x, y)$-plane. If a line $l^{\prime} \subset \mathbb{R}^{3} \backslash l_{z}$ projects down to a line $\pi\left(l^{\prime}\right)=\left\{(x, y) \in \mathbb{R}^{2} \mid A x+B y+1=0\right\}$, then $l^{\prime}$ can be uniquely represented as the intersection of the vertical plane

$$
p(A, B):=\left\{(x, y, z) \in \mathbb{R}^{3} \mid A x+B y+1=0\right\}
$$

and a plane $q(C, D):=\left\{(x, y, z) \in \mathbb{R}^{3} \mid C x+D y=z\right\}$ where $C$ and $D$ are suitable parameters. Every point $(x, y)$ on $\pi\left(l^{\prime}\right)$ lifts to the point $(x, y, C x+D y)$ on $l^{\prime}$. By definition the plane $q(C, D)$ contains the origin. We can read off the above-below relations of an arrangement $\mathscr{L}^{\prime}:=\left(l_{1}^{\prime}, \ldots, l_{n}^{\prime}\right)$ with respect to the projection $\pi$ by comparing the heights of the lines over the points of intersection in the projection $\pi\left(\mathscr{L}^{\prime}\right)$. For this we represent the lines $l_{i}^{\prime}$ by parameters $A_{i}, B_{i}, C_{i}$, and $D_{i}$ as described above. For two lines $l_{i}^{\prime}$ and $l_{j}^{\prime}$ we now define

$$
\Delta_{\mathscr{L}}(i, j)=\operatorname{sign}\left(C_{i} x_{i j}+D_{i} y_{i j}-C_{j} x_{i j}-D_{j} y_{i j}\right)
$$

where $\left(x_{i j}, y_{i j}\right)$ is the point of intersection of $\pi\left(l_{i}\right)$ and $\pi\left(l_{j}\right)$ in the $(x, y)$-plane. The map $\Delta_{\mathscr{L}}$ represents the above-below relations of $\mathscr{L}^{\prime}$ induced by $\pi$.

Definition 1.3. An affine weaving diagram $\mathscr{W}:=(\chi, \Delta)$ is called liftable, if a spatial line arrangement $\mathscr{L}^{\prime}$ such that $\chi_{x\left(x\left(\mathscr{L}^{\prime}\right)\right)}=\chi$ and $\Delta_{\mathscr{L}^{\prime}}(i, j)=\Delta(i, j)$ for

$$
(i, j) \in \Delta^{-1}(\{-1,0,1\})
$$

exists. 
We now see how the property that $\mathscr{L}^{\prime}$ is a lifting of $\mathscr{W}=(\chi, \Delta)$ can be expressed in terms of the realizability of oriented matroids. We consider an oriented matroid $\chi_{*}$ which describes the main combinatorial intent of $\mathscr{L}^{\prime}$. As described above the lines $l_{i}^{\prime}$ of $\mathscr{L}^{\prime}$ have a unique representation as intersections of planes $p\left(A_{i}, B_{i}\right)$ and $q\left(C_{i}, D_{i}\right)$. As we did for the planar case, we can consider these generating planes (represented by homogeneous coordinates) together with the plane at infinity as describing an oriented matroid. We define

$$
Y\left(\mathscr{L}^{\prime}\right):=\left(\begin{array}{ccrc}
A_{1} & B_{1} & 0 & 0 \\
C_{1} & D_{1} & -1 & 0 \\
A_{2} & B_{2} & 0 & 1 \\
C_{2} & D_{2} & -1 & 0 \\
\vdots & \vdots & \vdots & \vdots \\
A_{n} & B_{n} & 0 & 1 \\
C_{n} & D_{n} & -1 & 0 \\
0 & 0 & 0 & 1
\end{array}\right) .
$$

The oriented matroid $\chi_{Y\left(\mathscr{L}^{\prime}\right)}$ contains all information that is necessary to decide whether $\mathscr{L}^{\prime}$ is a lifting of $\mathscr{W}$. We assume that $\chi_{Y\left(\mathscr{L}^{\prime}\right)}$ is indexed by

$$
F:=\left\{1,1^{\prime}, 2,2^{\prime}, \ldots, n, n^{\prime}, n+1\right\}
$$

where $i$ is the index of the $(2 i-1)$ th row of $Y\left(\mathscr{L}^{\prime}\right)$ and $i^{\prime}$ is the index of the $2 i$ th row of $Y\left(\mathscr{L}^{\prime}\right)$. Furthermore, we set $E^{\prime}:=\left\{1^{\prime}, \ldots, n^{\prime}\right\}$. So we have

$$
F=E \uplus E^{\prime} \uplus\{n+1\} .
$$

Theorem 1.4. $\mathscr{L}^{\prime}$ is a lifting of $\mathscr{W}=(\chi, \Delta)$ if and only if $\chi_{Y\left(\mathscr{L}^{\prime}\right)}$ satisfies the following conditions:

(i) $\chi_{Y\left(\mathscr{L}^{\prime}\right)}\left(i, j, k, l^{\prime}\right)=\chi(i, j, k)$ for all $(i, j, k) \in(E \cup\{n+1\})^{3}$ and $l^{\prime} \in E^{\prime}$.

(ii) $\chi_{Y\left(\mathscr{L}^{\prime}\right)}\left(i, j, i^{\prime}, j^{\prime}\right)=\chi(i, j, n+1) \cdot \Delta(i, j)$ for all $(i, j) \in \Delta^{-1}(\{-1,0,1\})$.

Proof. We prove that condition (i) is equivalent to $\chi_{X\left(\pi\left(\mathscr{L}^{\prime}\right)\right)}=\chi$ and that condition (ii) is equivalent to $\Delta_{\mathscr{L}}(i, j)=\Delta(i, j)$ for $(i, j) \in \Delta^{-1}(\{-1,0,1\})$. Both facts together imply the theorem.

For the first fact we only have to prove that

$$
\chi_{\mathbf{Y}\left(\mathscr{L}^{\prime}\right)}\left(i, j, k, l^{\prime}\right)=\chi_{\mathbf{X}\left(\pi\left(\mathscr{L}^{\prime}\right)\right)}(i, j, k)
$$

holds for all $(i, j, k) \in(E \cup\{n+1\})^{3}$ and $l^{\prime} \in E^{\prime}$. This fact is immediate by observing 
that

$$
\operatorname{det}\left(\begin{array}{rrrr}
A_{i} & B_{i} & 0 & 1 \\
A_{j} & B_{j} & 0 & 1 \\
A_{k} & B_{k} & 0 & 1 \\
C_{l} & D_{l} & -1 & 0
\end{array}\right)=\operatorname{det}\left(\begin{array}{ccc}
A_{i} & B_{i} & 1 \\
A_{j} & B_{j} & 1 \\
A_{k} & B_{k} & 1
\end{array}\right)
$$

Here we assumed that the plane at infinity is given as $p\left(A_{n+1}, B_{n+1}\right)$ with parameters $A_{n+1}=B_{n+1}=0$.

For the second fact it is sufficient to prove that

$$
\chi_{Y\left(\mathscr{L}^{\prime}\right)}\left(i, j, i^{\prime}, j^{\prime}\right)=\chi(i, j, n+1) \cdot \Delta_{\mathscr{L}^{\prime}}(i, j)
$$

holds for all $(i, j) \in \Delta^{-1}(\{-1,0,1\})$. We observe

$$
\operatorname{det}\left(\begin{array}{rrrr}
A_{i} & B_{i} & 0 & 1 \\
A_{j} & B_{j} & 0 & 1 \\
C_{i} & D_{i} & -1 & 0 \\
C_{j} & D_{j} & -1 & 0
\end{array}\right)=\left(C_{i}-C_{j}\right) \cdot\left(B_{i}-B_{j}\right)+\left(D_{i}-D_{j}\right) \cdot\left(A_{j}-A_{i}\right)
$$

The intersection of the lines

$$
\pi\left(l_{i}\right):=\left\{(x, y) \mid A_{i} x+B_{i} y+1=0\right\}
$$

and

$$
\pi\left(l_{j}\right):=\left\{(x, y) \mid A_{j} x+B_{i} y+1=0\right\}
$$

is the point

$$
\left(x_{i j}, y_{i j}\right)=\left(\frac{B_{i}-B_{j}}{A_{i} B_{j}-B_{i} A_{j}}, \frac{A_{j}-A_{i}}{A_{i} B_{j}-B_{i} A_{j}}\right) .
$$

Combining (*) and (**) with the definition of

$$
\Delta_{\mathscr{L}}(i, j):=\operatorname{sign}\left(\left(C_{i}-C_{j}\right) x_{i j}+\left(D_{i}-D_{j}\right) y_{i j}\right)
$$

we obtain

$$
\Delta_{\mathscr{L}^{\prime}}(i, j) \cdot \operatorname{sign}\left(A_{i} B_{j}-A_{j} B_{i}\right)=\chi_{Y\left(\mathscr{C}^{\prime}\right)}\left(i, j, i^{\prime}, j^{\prime}\right)
$$


Applying the identity

$$
\operatorname{sign}\left(A_{i} B_{j}-A_{j} B_{i}\right)=\operatorname{sign} \operatorname{det}\left(\begin{array}{ccc}
A_{i} & B_{i} & 0 \\
A_{j} & B_{j} & 0 \\
0 & 0 & 1
\end{array}\right)=\chi(i, j, n+1)
$$

to $(* * *)$ finally proves the claim.

We have seen that the fact that $\mathscr{L}^{\prime}$ is a lifting of $\mathscr{W}$ can be expressed by sign conditions of the underlying oriented matroid. This allows us to express the liftability of $\mathscr{W}$ as the realizability of a certain partial oriented matroid.

Definition 1.5. The lifting oriented matroid $\chi_{\Psi}$ of an affine weaving diagram $\mathscr{W}=(\chi, \Delta)$ on $E$ is a partial oriented matroid on $E \uplus E^{\prime} \uplus\{n+1\}$ of rank 4 defined by

$$
\chi_{\mathscr{W}}(i, j, k, l):= \begin{cases}0 & \text { if }(i, j, k, l) \in(E \uplus\{n+1\})^{4} \uplus\left(E^{\prime}\right)^{4} \\ \chi(i, j, k) & \text { if }(i, j, k) \in(E \cup\{n+1\})^{3} \text { and } l \in E^{\prime} \\ \chi(i, j, n+1) \cdot \Delta(i, j) & \text { if }(i, j) \in E^{2} \text { and } k=i^{\prime}, \quad l=j^{\prime} \\ * & \text { otherwise. }\end{cases}
$$

It can be easily checked that by this definition $\chi_{\mathscr{W}}$ satisfies the requirements of Definition 1.1 to be a partial oriented matroid. As a consequence of Theorem 1.4 we obtain:

Corollary 1.6. The lifting oriented matroid $\chi_{\mathscr{W}}$ is realizable if and only if $\mathscr{W}$ is liftable.

Proof. For every spatial line arrangement $\mathscr{L}^{\prime}:=\left(l_{1}^{\prime}, \ldots, l_{n}^{\prime}\right)$ the oriented matroid $\chi_{Y\left(\mathscr{E}^{\prime}\right)}$ defined above satisfies

$$
\chi_{\mathbf{Y}\left(\mathscr{L}^{\prime}\right)}(i, j, k, l)=0
$$

for any $(i, j, k, l) \in(E \uplus\{n+1\})^{4} \cup\left(E^{\prime}\right)^{4}$, since the submatrices $\left(Y\left(\mathscr{L}^{\prime}\right)_{i}\right)_{i \in E \cup\{n+1\}}$ and $\left(Y\left(\mathscr{L}^{\prime}\right)_{i}\right)_{i \in E^{\prime}}$ both have rank 3 . If $\mathscr{L}^{\prime}$ is a lifting of $\mathscr{W}$, then $\chi_{Y\left(\mathscr{L}^{\prime}\right)}$ by Theorem 1.4 also satisfies the second and third cases of Definition 1.5. Therefore $Y\left(\mathscr{L}^{\prime}\right)$ is a realization of the lifting oriented matroid $\chi_{W}$.

Conversely, if $Y$ is a realization of $\chi_{\psi}$, then all planes corresponding to indices $E \uplus\{n+1\}$ meet in a single point, and so do all planes corresponding to indices $E^{\prime}$. Applying a suitable linear transformation of $Y$ by multiplying with a regular $4 \times 4$ matrix $T$ with positive determinant we can achieve that $Y \cdot T$ has the form of a matrix $Y\left(\mathscr{L}^{\prime}\right)$ for some spatial line arrangement $\mathscr{L}^{\prime}$. Since the linear transformation preserves the underlying oriented matroid $Y \cdot T=Y\left(\mathscr{L}^{\prime}\right)$ is still a realization of $\chi_{\mathscr{W}}$. By Theorem 1.4 we conclude that $\mathscr{L}^{\prime}$ is a lifting of $\mathscr{W}$. 
Now Corollary 1.6 allows us to consider the problem of liftability as a special case of the realizability problem for (partial) oriented matroids. This way the liftability problem can be treated by standard methods of proving nonrealizability for oriented matroids, as presented in [5], [7], [8], and [19]. In principle the nonliftability of an affine weaving diagram $\mathscr{W}=(\chi, \Delta)$ can have two different reasons. One reason is that the rank 3 oriented matroid $\chi$ can be nonrealizable. In this case there is no spatial line arrangement $\mathscr{L}^{\prime}$ projecting down on a line arrangement corresponding to $\chi$. In this case the property of $\mathscr{W}$ not being liftable is independent of the special above-below relations indicated by $\Delta$. The second reason for nonliftability is that for every spatial line arrangement $\mathscr{L}^{\prime}$ projecting to a line arrangement compatible with $\chi$ the above-below relations $\Delta_{\mathscr{L}}$ are not compatible with the above-below relations indicated by $\Delta$. In the latter case the nonliftability is a proper spatial property. We analyze such situations in the next two sections.

Up to now we presented the theory of line arrangements in the plane and their liftings in a mainly affine setting. We did this by adding special "external" constructions that encode the special affine setting: Whenever we considered planar line arrangements we added a line at infinity (represented by homogeneous coordinates $(0,0,1))$ to the arrangement encoding the affine structure of the arrangement. Likewise we described the connection of the lifted and the planar line arrangement by a special orthogonal projection $\pi$.

We now consider an intrinsic setting of weaving diagrams (indicated by dropping the adjective "affine") that allows us to get rid of these external elements. We thereby loose the control on the special affine situation (we get some kind of oriented projective setting, see [22] and [23]) but we gain a more elegant setting for the theory. The structure to be described is essentially the same as the "link chirotopes" presented in [9]. We describe this setting in similar terms as the affine case.

We again consider an arrangement $\mathscr{L}:=\left(l_{1}, \ldots, l_{n}\right)$ of oriented lines not containing the origin in the plane $\mathbb{R}^{2}$. We may assume that the origin lies on the same side of each of the lines (either the positive or the negative). The matrix $\hat{X}(\mathscr{L})$, where the rows are these homogeneous coordinates of the lines, again defines an acyclic oriented matroid $\chi_{\left.\ell_{(} \mathscr{L}^{\prime}\right)}$ on $E:=\{1, \ldots, n\}$. Since $\chi_{\mathcal{P}_{\left(\mathscr{L}^{\prime}\right)}}$ does not contain information on the position of the line at infinity, it is invariant under projective moves of the line arrangement. If $l_{i} \cap l_{j}$ is an extreme vertex of the arrangement, the oriented matroid does not change, if we move $l_{i}$ and $l_{j}$ in such a way that $l_{i} \cap l_{j}$ "passes" infinity and "comes back" at the opposite end of the arrangement, while nothing else changed (see Fig. 2$)$. We call such a move an $(i, j)$-infinity move.

We now encode the above-below choices for $\mathscr{L}$ in terms of a linking coefficient $\Lambda(i, j)$ for $(i, j) \in E^{2} \backslash \bar{E} . \Lambda: E^{2} \backslash \bar{E} \rightarrow\{-1,0,1, *\}$ is an alternating function taking the value +1 if the upper line must rotate counterclockwise (by an angle of less than $\left.180^{\circ}\right)$ to match the lower line in the same orientation. $\Lambda(i, j)$ is negative if the rotation is clockwise. The values " 0 " and "*" correspond to the incident and the "don't care" cases, respectively. Notice that for a given picture the function $\Lambda(i, j)$ is symmetrical in $i$ and $j$. 


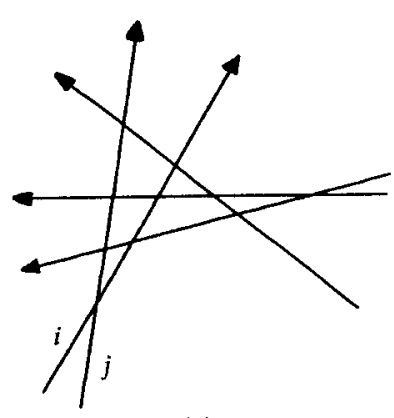

(a)

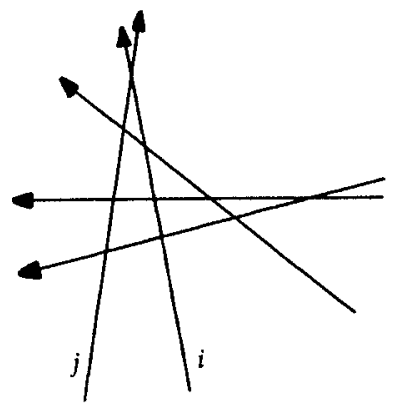

(b)

Fig. 2. Infinity moves in arrangements of pseudolines.

The linking coefficient has a natural interpretation as an invariant of two oriented skew lines in 3-space. If the lines $l_{i}$ and $l_{j}$ are the projections of lines $l_{i}^{\prime}=\overrightarrow{a_{i} b_{i}} \in \mathbb{R}^{3}$ and $l_{j}^{\prime}=\overrightarrow{a_{j} b_{j}} \in \mathbb{R}^{3}$ directed from $a_{i}$ to $b_{i}$ and $a_{j}$ to $b_{j}$, respectively, then the linking coefficient $\Lambda(i, j)$ represents the orientation of the simplex

$$
\left(a_{i}, a_{j}, b_{i}, b_{j}\right)
$$

The sign is independent of the chosen projection and the special choice of $a_{i}, a_{j}, b_{i}$, and $b_{j}$.

Definition 1.7. A link diagram $\mathscr{D}:=(\chi, \Lambda)$ on $E:=\{1, \ldots, n\}$ consists of an acyclic uniform oriented matroid $\chi$ on $E$ and a symmetric mapping

$$
\Lambda: E^{2} \backslash \bar{E} \rightarrow\{-1,0,+1, *\} .
$$

$\chi$ is called the arrangement of $\mathscr{D}$ and $\Lambda$ is called the weaving pattern of $\mathscr{D} . \mathscr{D}$ is called complete if $\operatorname{Im}(\Lambda) \subseteq\{-1,0,+1\}$. D is called uniform if $\operatorname{Im}(\Lambda) \subseteq\{-1,+1\}$.

We see that for every affine weaving diagram $\mathscr{W}=(\chi, \Delta)$ we can obtain a corresponding link diagram $\mathscr{D}_{\mathscr{W}}:=\left(\chi^{\prime}, \Lambda\right)$ by setting $\chi^{\prime}=\chi \backslash\{n+1\}$ and $\Lambda(i, j):=$ $\Delta(i, j) \cdot \chi(i, j, n+1)$. The reader may easily convince himself that this choice is compatible with the two different interpretations of a planar picture as an affine weaving diagram and as a link diagram. However, different affine weaving diagrams may translate to the same link diagram. Two affine weaving diagrams $\mathbb{W}_{1}$ and $\mathscr{W}_{2}$ generate the same link diagram if and only if there is a sequence of affine weaving diagrams

$$
\mathscr{W}_{1}:=\mathscr{W}^{1}=\left(\chi^{1}, \Delta^{1}\right), \mathscr{W}^{2}=\left(\chi^{2}, \Delta^{2}\right), \ldots, \mathscr{W}^{k}=\left(\chi^{k}, \Delta^{k}\right)=: \mathscr{W}_{2}
$$

such that the corresponding pictures of $\mathscr{W}^{d}$ and $\mathscr{W}^{d+1}$ for $l \in\{1, \ldots, k-1\}$ are 


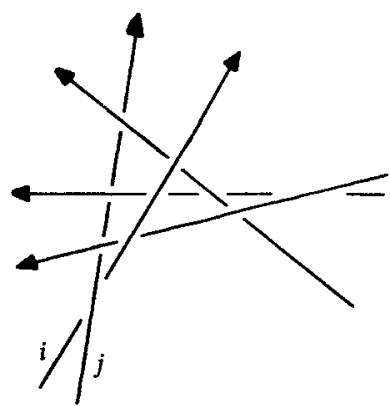

(a)

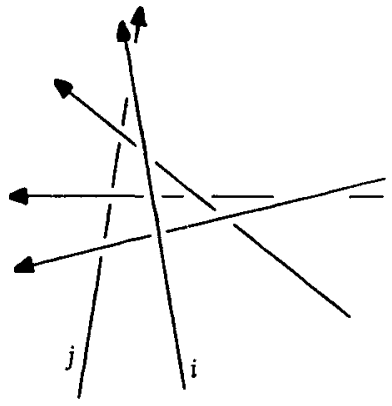

(b)

Fig. 3. Infinity moves in link diagrams. In both parts $\Lambda(i, j)=-1$.

connected by a $(i, j)$-infinity move and $\Delta^{l}(i, j)=-\Delta^{l+1}(i, j)$. In other words, moving a vertex through infinity and changing the above-below relation preserves the linking coefficient (see Fig. 3).

Similar to the affine case we can define a lifting chirotope by:

Definition 1.8. The lifting oriented matroid $\chi_{\mathscr{D}}$ of a link diagram $\mathscr{D}=(\chi, \Lambda)$ on $E$ is a partial oriented matroid on $E \uplus E^{\prime}$ of rank 4 defined by

$$
\chi_{\mathscr{D}}(i, j, k, l):= \begin{cases}0 & \text { if }(i, j, k, l) \in(E)^{4} \cup\left(E^{\prime}\right)^{4}, \\ \chi(i, j, k) & \text { if }(i, j, k) \in(E)^{3} \text { and } l \in E^{\prime} \\ \Lambda(i, j) & \text { if }(i, j) \in E^{2} \text { and } k=i^{\prime}, l=j^{\prime} \\ * & \text { otherwise. }\end{cases}
$$

A link diagram $\mathscr{D}$ is called liftable if $\chi_{\mathscr{D}}$ is realizable.

This way, for an affine weaving diagram $\mathscr{W}$ with corresponding link diagram $\mathscr{D}$, we have $\chi_{\mathscr{W}} \backslash\{n+1\}=\chi_{\mathscr{D}}$. The nonliftability of an affine weaving diagram is mostly a consequence of the nonliftability of the underlying link diagram.

\section{How To Make Lifting Oriented Matroids Rigid}

Clearly, the above constructions of lifting oriented matroids for link diagrams and weaving diagrams still carry a lot of freedom. There may be many liftings $\mathscr{L}^{\prime}$ compatible with a given diagram, each resulting in a different corresponding oriented matroid. This is reflected by the fact that the lifting oriented matroid is by definition partial.

In the next section we need a way to derive a proper (i.e., nonpartial) oriented matroid that contains the lifting oriented matroid as a substructure. The method presented to solve this problem is more geometric than combinatoric in nature and it does not easily generalize to the underlying oriented matroid. We restrict 
ourselves to liftings that project down to a concrete line arrangement $\mathscr{L}$ (given by coordinates rather than by its oriented matroid). The existence of such particular liftings implies the existence of a particular oriented matroid.

Again let $\mathscr{L}:=\left(l_{1}, \ldots, l_{n}\right)$ be a line arrangement in $\mathbb{R}^{2}$ given by homogeneous coordinates $\left(A_{i}, B_{i}, 1\right)_{i \in E}$ and let $\chi:=\chi_{\mathcal{R}_{(\mathscr{L})}}$ be the corresponding oriented matroid. We assume that the origin $o$ is chosen in such a way that it does not meet one of the lines. Furthermore, we assume that no pair of points of intersection of the lines in $\mathscr{L}$ are collinear with the origin. The last assumption is crucial for our construction.

Let

$$
p_{i j}:=l_{i} \wedge l_{j}=\left(\begin{array}{c}
A_{i} \\
B_{i} \\
1
\end{array}\right) \times\left(\begin{array}{c}
A_{j} \\
B_{j} \\
1
\end{array}\right)=\left(\begin{array}{c}
B_{i}-B_{j} \\
A_{j}-A_{i} \\
A_{i} B_{j}-A_{j} B_{i}
\end{array}\right)
$$

be the homogeneous coordinates for the points of intersection of two lines.

Let $\mathscr{D}=(\chi, \Lambda)$ be a link diagram on $\mathscr{L}$. Assume that there is a lifting $\mathscr{L}^{\prime}:=\left(l_{1}^{\prime}, \ldots, l_{n}^{\prime}\right) \subset \mathbb{R}^{3}$ of $\mathscr{D}=(\chi, \Delta)$ projecting down exactly on the positions of the lines in $\mathscr{L}$. In this case we have a unique representation of the $l_{i}^{\prime}$ given by $p\left(A_{i}, B_{i}\right) \cap q\left(C_{i}, D_{i}\right)$ as described above. We now consider the matrix

$$
Y_{\varepsilon}:=Y_{\varepsilon}\left(\mathscr{L}^{\prime}\right):=\left(\begin{array}{ccrc}
A_{1} & B_{1} & 0 & 1 \\
A_{1}+\varepsilon C_{1} & B_{1}+\varepsilon D_{1} & -1 & 0 \\
A_{2} & B_{2} & 0 & 1 \\
A_{2}+\varepsilon C_{2} & B_{2}+\varepsilon D_{2} & -1 & 0 \\
\vdots & \vdots & \vdots & \vdots \\
A_{n} & B_{n} & 0 & 1 \\
A_{n}+\varepsilon C_{n} & B_{n}+\varepsilon D_{n} & -1 & 0
\end{array}\right) .
$$

Canonically we identify $Y_{\varepsilon}$ with a line arrangement $\mathscr{L}_{\varepsilon}^{\prime}$ in $\mathbb{R}^{3}$ given by the lines $p\left(A_{i}, B_{i}\right) \cap q\left(A_{i}+\varepsilon C_{i}, B_{i}+\varepsilon D_{i}\right)$.

\section{Lemma 2.1 .}

(i) For every $\varepsilon>0$ the matrix $Y_{\varepsilon}$ represents a lifting $\mathscr{L}_{\varepsilon}^{\prime}$ of $\mathscr{D}$.

(ii) With the above choices of $\mathscr{L}, \mathscr{D}$, and o the oriented matroid $\chi_{Y_{\varepsilon}}$ is uniquely determined if $\varepsilon>0$ is sufficiently small.

Proof. The first fact is a direct consequence of the following identity:

$$
\operatorname{det}\left(\begin{array}{ccrr}
A_{i} & B_{i} & 0 & 1 \\
A_{j} & B_{j} & 0 & 1 \\
A_{i}+\varepsilon C_{i} & B_{i}+\varepsilon D_{i} & -1 & 0 \\
A_{j}+\varepsilon C_{j} & A_{j}+\varepsilon D_{j} & -1 & 0
\end{array}\right)=\varepsilon \cdot \operatorname{det}\left(\begin{array}{cccc}
A_{i} & B_{i} & 0 & 1 \\
A_{j} & B_{j} & 0 & 1 \\
C_{i} & D_{i} & -1 & 0 \\
C_{j} & D_{j} & -1 & 0
\end{array}\right)
$$

which can be easily checked. 
To prove the second fact, we directly derive the sign pattern of the "limit oriented matroid" $\chi_{0}:=\chi[\mathscr{L}, o, \mathscr{D}]=\lim _{\varepsilon \rightarrow 0}\left(\chi_{Y_{2}}\right)$. We compute $\chi_{0}(i, j, k, l)$ for the different choices of $(i, j, k, l) \in\left(E \cup E^{\prime}\right)^{4}$. Since by (i) $\chi_{0}$ already contains the lifting oriented matroid $\chi_{\mathscr{D}}$ of $\mathscr{D}$ as a substructure, only a few cases are left.

Case $1: i \in E ; j, k, l \in E$. We get

$$
\begin{aligned}
\chi_{0}(i, j, k, l) & :=\operatorname{sign} \lim _{\varepsilon \rightarrow 0} \operatorname{det}\left(\begin{array}{ccrr}
A_{i} & B_{i} & 0 & 1 \\
A_{j}+\varepsilon C_{j} & B_{j}+\varepsilon D_{j} & -1 & 0 \\
A_{k}+\varepsilon C_{k} & B_{k}+\varepsilon D_{k} & -1 & 0 \\
A_{l}+\varepsilon C_{l} & A_{l}+\varepsilon D_{l} & -1 & 0
\end{array}\right) \\
& =\operatorname{sign} \lim _{\varepsilon \rightarrow 0} \operatorname{det}\left(\begin{array}{lll}
A_{j}+\varepsilon C_{j} & B_{j}+\varepsilon D_{j} & -1 \\
A_{k}+\varepsilon C_{k} & B_{k}+\varepsilon D_{k} & -1 \\
A_{l}+\varepsilon C_{l} & A_{l}+\varepsilon D_{l} & -1
\end{array}\right) \\
& =\chi(j, k, l) .
\end{aligned}
$$

The last identity holds since $\chi(i, j, k) \neq 0$ and therefore for sufficiently small $\varepsilon$ the coefficients $C_{j}, C_{k}, C_{l}, D_{j}, D_{k}$, and $D_{l}$ do not influence the sign of the determinant.

Case 2: $i, j \in E ; k, l \in E^{\prime}$. The case $\left\{i^{\prime}, j^{\prime}\right\}=\{k, j\}$ is already covered by our considerations of statement (i). Therefore we restrict ourselves to the case $i^{\prime} \neq k$, $i^{\prime} \neq l$. We get

$$
\begin{aligned}
\chi_{0}(i, j, k, l):=\operatorname{sign} \lim _{\varepsilon \rightarrow 0} \operatorname{det}\left(\begin{array}{cccc}
A_{i} & B_{i} & 0 & 1 \\
A_{j} & B_{j} & 0 & 1 \\
A_{k}+\varepsilon C_{k} & B_{k}+\varepsilon D_{k} & -1 & 0 \\
A_{l}+\varepsilon C_{l} & A_{l}+\varepsilon D_{i} & -1 & 0
\end{array}\right) \\
=\operatorname{sign}\left(\left(A_{k}-A_{l}\right)\left(B_{j}-B_{j}\right)+\left(B_{k}-B_{l}\right)\left(A_{j}-A_{i}\right)\right. \\
\left.+\lim _{\varepsilon \rightarrow 0}\left(\varepsilon \cdot\left(C_{k}-C_{l}\right)\left(B_{j}-B_{j}\right)+\varepsilon \cdot\left(D_{k}-D_{l}\right)\left(A_{j}-A_{k}\right)\right)\right)
\end{aligned}
$$

If $\varepsilon$ is sufficiently small and $\alpha:=\left(A_{k}-A_{l}\right)\left(B_{j}-B_{j}\right)+\left(B_{k}-B_{l}\right)\left(A_{j}-A_{j}\right) \neq 0$, then we obtain $\chi_{0}(i, j, k, l)=\operatorname{sign}(\alpha)$. The fact $\alpha \neq 0$ is a direct consequence of our choice of the origin. We get

$$
\alpha:=\operatorname{det}\left(p_{k l}, p_{i j}, o\right)=\left(l_{k} \wedge l_{l}\right) \vee\left(l_{i} \wedge l_{j}\right) \vee o
$$

In all cases the derived signs are independent of the special choice of the $C_{i}$, $D_{i}$. This proves the claim.

As a direct consequence of this construction we obtain: 
Theorem 2.2. If $\mathscr{D}$ is liftable to an arrangement $\mathscr{L}^{\prime}$ which satisfies $\pi\left(\mathscr{L}^{\prime}\right)=\mathscr{L}$, then the oriented matroid $\chi[\mathscr{L}, o, \mathscr{D}]$ as described above is realizable.

\section{Cycling in Weavings and Oriented Matroids}

In this section we describe the connection between noneuclideanness for oriented matroids and a certain kind of nonliftability proof for link diagrams. Roughly speaking an oriented matroids is called euclidean if it fulfills a certain combinatoric variant of Euclid's postulate of the parallels (see [14]). Here we use a different approach due to Fukuda and Mandel [12], [14] that defines euclideanness as the nonexistence of cycles in the oriented matroid equivalent of linear programming [4]. For an extensive introduction in oriented matroid programming and euclideanness of oriented matroids the reader is referred to [4], [3, Chapter 10], [12], and [14]. Usually euclideanness is defined in terms of properties on the set of covectors of an oriented matroid. Here we define euclideanness directly in terms of the bases orientations of the oriented matroid. The equivalence of this characterization to the one given by Fukuda and Mandel is due to the author and can be found in [19] or [21]. For our purposes only the rank 4 case is important and to avoid unnecessary technicalities we restrict our setting to this case. Unless otherwise stated we now assume that $\chi$ is of rank 4 .

Definition 3.1. A triple $(\chi, g, f)$ where $\chi$ is a (rank 4) partial oriented matroid on $E \uplus\{f, g\}$ and $f, g$ are neither loops nor coloops in $\chi$ is called an oriented matroid program. A 3-set $\{a, b, c\} \subset E$ is called a base of $(\chi, g, f)$ if $\chi(a, b, c, g) \neq 0$. An ordered pair of bases $\{a, b, c\} \rightarrow\{a, b, d\}$ that differ in exactly one element is called a pivot operation. A privot operation $\{a, b, c\} \rightarrow\{a, b, d\}$ is called:

degenerate if $\chi(a, b, c, d)=0$,

horizontal if $\chi(a, b, f, g)=0$,

strictly increasing if $\chi(a, b, c, d) \cdot \chi(a, b, g, d) \cdot \chi(a, b, g, d) \cdot \chi(a, b, g, f)=+1$, strictly decreasing if $\chi(a, b, c, d) \cdot \chi(a, b, g, d) \cdot \chi(a, b, g, d) \cdot \chi(a, b, g, f)=-1$.

To see the relation of this definition to linear programming consider an affine arrangement $\mathscr{H}$ of $n+2$ oriented hyperplanes $1, \ldots, n, f, g$ in $\mathbb{R}^{3}$ given by homogenous coordinates $X:=\left(h_{1}, \ldots, h_{n}, h_{f}, h_{g}\right)^{T}$ where $h_{g}=(0,0,0,1)$ plays the role of the plane at infinity. For the oriented matroid $\chi_{X}$, we get the following interpretation of the above definition: The bases of $\left(\chi_{x}, g, f\right)$ correspond to triples of planes indexed by elements of $E$ intersecting properly in only one point. The lines of $\mathscr{H}$ are the proper intersections of a pair of planes $(a, b) \in E^{2}$. A pivot operation $B_{1}:=\{a, b, c\} \rightarrow\{a, b, d\}=: B_{2}$ is a pair of bases joined by a line (the intersection of plane $a$ and $b$ ). The oriented plane $f$ induces an orientation on each of the lines. A pivot operation $B_{1} \rightarrow B_{2}$ is degenerate if the points corresponding to $B_{1}, B_{2}$ are identical. It is horizontal if the line joining the points is parallel to $f$. It is strictly increasing if the line $a \cap b$ points from $B_{1}$ to $B_{2}$ (according to the direction of $a \cap b$ induced by $f$ ). 
Using these definitions we are able to encode linear programming problems purely on the level of oriented matroid programs. Here $f$ plays the role of the objective function, $g$ defines the particular affine situation, and $1, \ldots, n$ describe the hyperplanes corresponding to the inequalities of the linear programming problem. In the realizable case the strictly increasing pivot operations correspond to bases-changes with increasing objective function. For horizontal and degenerate pivot operations the value of the objective function remains unchanged. The euclidean oriented matroid programs are those where a nondegenerate pivot cycling cannot occur:

Definition 3.2. An oriented matroid program $(\chi, g, f)$ is called noneuclidean if there is a cyclic sequence of pivot operations,

$$
B_{1} \rightarrow B_{2} \rightarrow \cdots \rightarrow B_{k} \rightarrow B_{1}
$$

such that every pivot operation is either horizontal, degenerate, or strictly increasing and at least one pivot operation is strictly increasing. Otherwise $(\chi, g, f)$ is called euclidean. If $(\chi, g, f)$ is noneuclidean, then $\chi$ is also called noneuclidean.

Clearly, all oriented matroid programs arising from realizable situations are euclidean, since in such a case no nondegenerate cycling can occur (see Lemma 10.1.17 of [3]). As a direct consequence of this fact we obtain:

Corollary 3.3. If $(\chi, g, f)$ is noneuclidean, then $\chi$ is nonrealizable.

We now see that there is a very close connection between a structure of the link or weaving diagram, and the euclideanness of the corresponding lifting oriented matroid. To avoid unnecessary technical difficulties we consider only the case of uniform link and weaving diagrams. Similarly to the oriented matroid case, a link program is a pair $(\mathscr{D}, i)$ where $\mathscr{D}=(\chi, \Lambda)$ is a uniform link diagram on $E$ and $i \in E$. As we will see later, the line $l_{i}$ simultaneously plays the role of $f$ and $g$ in a corresponding oriented matroid program. We can always assume that $\mathscr{D}$ is given in the form of a particular uniform weaving diagram (i.e., a special picture) $\mathscr{W}$ with $\mathscr{D}=\mathscr{D}_{\mathscr{W}}$. The pair $(\mathscr{W}, i)$ is also called a link program.

Definition 3.4. A link program $(\mathscr{W}, i)$ is called noneuclidean if there exists a sequence $\lambda_{1}, \ldots, \lambda_{k} \in E \backslash\{i\}$ such that for $1 \leq j \leq k$ the above-below relations of the points

$$
l_{\lambda_{j}} \cap l_{i}, \quad l_{\lambda_{j}} \cap l_{\lambda_{j-i}}, \quad l_{\lambda_{j}} \cap l_{\lambda_{j+1}}
$$

occur in alternating order while passing along line $l_{\lambda_{j}}$ in positive direction (indices counted modulo $k$ ). 


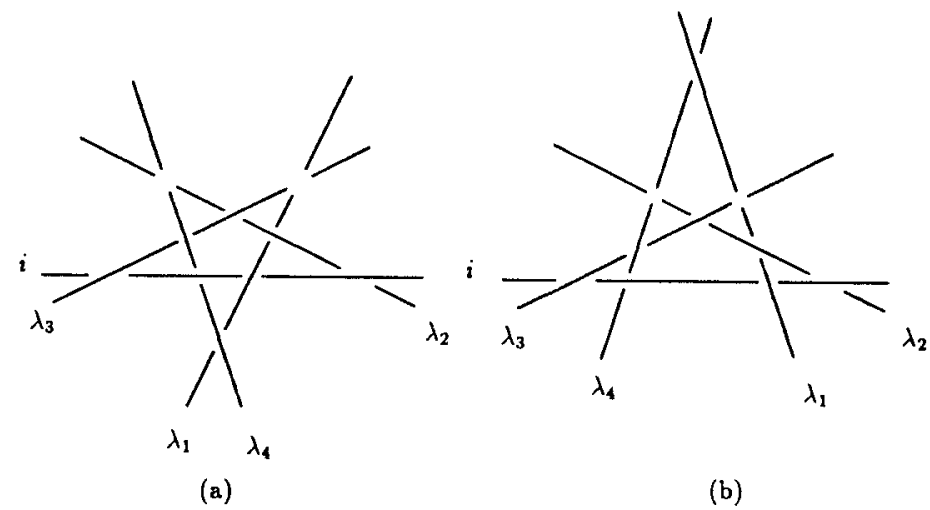

Fig. 4. Noneuclidean link diagrams.

Figure 4(a) gives an example of a noneuclidean weaving diagram (only the relevant crossings are drawn). A sequence $\lambda_{1}, \ldots, \lambda_{k}$ satisfying the property described in the above definition is called a nondegenerate cycle in $(\mathscr{W}, i)$. In fact, the property of $(\mathscr{W}, i)$ being noneuclidean is invariant under infinity moves on $\mathscr{W}$. This is a consequence of the fact that the property "three above-below choices along one line alternate" is invariant under infinity moves. Figure 4 (b) arises from Fig. $4(\mathrm{a})$ after a $\left(\lambda_{1}, \lambda_{4}\right)$-infinity move is performed. This invariance of noneuclideanness under infinity moves justifies us to speak of a noneuclidean link program $(\mathscr{D}, i) .\left(\mathscr{D}_{\mathscr{W}}, i\right)$ is noneuclidean if and only if $(\mathscr{W}, i)$ is noneuclidean. We call a link diagram $\mathscr{D}$ on $E$ noneuclidean if there is an $i \in E$ such that $(\mathscr{D}, i)$ is noneuclidean.

The next lemma gives a characterization of how to discover three alternating crossings along one line directly from a link diagram. Applying this statement to Definition 3.4 we also immediately obtain an intrinsic characterization of noneuclidean link diagrams.

Lemma 3.5. Let $\mathscr{D}=(\chi, \Lambda)$ be a uniform link diagram, let $i \in E$, and let $a, b, c \in E \backslash i$ be pairwise different. The above-below relations of the intersections $l_{i} \cap l_{a}, l_{i} \cap l_{b}$, $l_{i} \cap l_{c}$ alternate along line $l_{i}$ if and only if the following three conditions are satisfied:

(i) $\chi(i, a, b) \cdot \chi(i, a, c) \cdot \Lambda(i, b) \cdot \Lambda(i, c)=-1$.

(ii) $\chi(i, b, c) \cdot \chi(i, b, a) \cdot \Lambda(i, c) \cdot \Lambda(i, a)=-1$.

(iii) $\chi(i, c, a) \cdot \chi(i, c, b) \cdot \Lambda(i, a) \cdot \Lambda(i, b)=-1$.

Proof. First notice that the statement that the above-below relations of the intersections $l_{i} \cap l_{a}, l_{i} \cap l_{b}, l_{i} \cap l_{c}$ alternate along line $l_{i}$ as well as the three conditions of the lemma taken together are invariant under the following three 


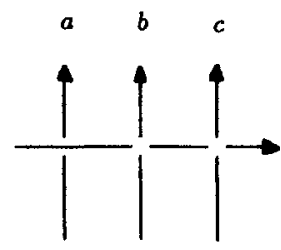

(a)

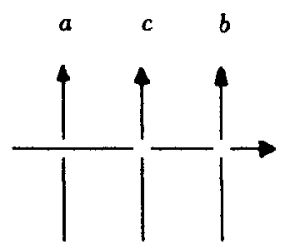

(b)

Fig. 5. The two cases in the proof of Lemma 3.5.

operations:

(1) Reversing the orientation of any of the lines $l_{i}, l_{a}, l_{b}, l_{c}$.

(2) Cyclic rotation of the indices $a, b, c$.

(3) Reversing simultaneously all above-below relations $\Lambda(i, a), \Lambda(i, b), \Lambda(i, c)$.

Hence, we may assume without loss of generality that we have a picture (a part of a corresponding weaving diagram $\mathscr{W}$ ) of the involved lines where $l_{i}$ points from the left to the right, the lines $l_{a}, l_{b}, l_{c}$ all point upward, and the sequence along the line $l_{i}$ starts with an undercrossing of line $l_{a}$ under $l_{i}$. The two possible cases are given in Fig. 5.

In case (a) we get

$$
\chi(i, a, b)=+1, \quad \chi(i, b, c)=+1, \quad \chi(i, a, c)=+1, \quad \Lambda(i, a)=+1 .
$$

If furthermore, we assume that the crossings alternate we get $\Lambda(i, b)=-1$ and $\Lambda(i, c)=+1$ and the three statements (i)-(iii) are satisfied. Conversely, if (i)-(iii) hold we can conclude $\Lambda(i, b)=-1$ and $\Lambda(i, c)=+1$ and obtain an alternating sequence.

A completely similar argument proves the equivalence in case (b).

Notice that by this lemma a nondegenerate cycle $\lambda_{1}, \ldots, \lambda_{k}$ can be completely rediscovered in the link program $(\mathscr{D}, i)$. We now see how noneuclideanness of a link diagram $\mathscr{D}$ carries over to the noneuclideanness of the corresponding lifting oriented matroid $\chi_{\mathscr{D}}$.

Theorem 3.6. If $(\mathscr{D}, i)$ is a noneuclidean link program, then the oriented matroid program $\left(\chi_{\mathscr{D}}, i, i^{\prime}\right)$ is also noneuclidean.

Proof. If $(\mathscr{D}, i)=((\chi, \Lambda), i)$ is noneuclidean, then by Definition 3.1 and Lemma 3.5 we have a sequence $\lambda_{1}, \ldots, \lambda_{k} \in E \backslash\{i\}$ of indices such that for each $1 \leq j \leq k$ we have:

(i) $)_{j} \chi\left(\lambda_{j}, i, \lambda_{j-1}\right) \cdot \chi\left(\lambda_{j}, i, \lambda_{j+1}\right) \cdot \Lambda\left(\lambda_{j}, \lambda_{j-1}\right) \cdot \Lambda\left(\lambda_{j}, \lambda_{j+1}\right)=-1$.

(ii) $)_{j}\left(\lambda_{j}, \lambda_{j-1}, \lambda_{j+1}\right) \cdot \chi\left(\lambda_{j}, \lambda_{j-1}, i\right) \cdot \Lambda\left(\lambda_{j}, \lambda_{j+1}\right) \cdot \Lambda\left(\lambda_{j}, i\right)=-1$.

(iii) ${ }_{j} \chi\left(\lambda_{j}, \lambda_{j+1}, i\right) \cdot \chi\left(\lambda_{j}, \lambda_{j+1}, \lambda_{j-1}\right) \cdot \Lambda\left(\lambda_{j}, i\right) \cdot \Lambda\left(\lambda_{j}, \lambda_{j-1}\right)=-1$. 
Here and in the rest of the proof we always assume that the indices are counted modulo $k$. For $1 \leq j \leq k$ we define $B_{j}:=\left\{\lambda_{j}, \lambda_{j}^{\prime}, \lambda_{j-1}\right\}$ and $B_{j}^{\prime}:=\left\{\lambda_{j}, \lambda_{j}^{\prime}, \lambda_{j+1}\right\}$. We prove that for $1 \leq j \leq k$ the pivot operations $B_{j} \rightarrow B_{j}^{\prime}$ and $B_{j}^{\prime} \rightarrow B_{j+1}$ are either all strictly increasing or all strictly decreasing in the oriented matroid program $\left(\chi_{\mathscr{P}}, i, i^{\prime}\right)$. If all those pivots are strictly increasing

$$
B_{1} \rightarrow B_{1}^{\prime} \rightarrow B_{2} \rightarrow B_{2}^{\prime} \rightarrow \cdots \rightarrow B_{k} \rightarrow B_{k}^{\prime} \rightarrow B_{1}
$$

forms a strictly increasing pivot cycle and proves the noneuclideanness of $\left(\chi_{\mathscr{D}}, i, i^{\prime}\right)$. In the second case the reversed order proves the noneuclideanness.

As we will see in the next paragraph, replacing $\mathscr{D}=(\chi, \Lambda)$ by $\mathscr{D}^{-}:=(\chi,-\Lambda)$ reverses the directions of all pivot operations in the oriented matroid program. Therefore we can assume that $B_{1} \rightarrow B_{1}^{\prime}$ is strictly increasing. From this and the noneuclideanness of $(\mathscr{D}, i)$ we have to conclude that the remaining pivot operations are also strictly increasing.

By Definition 3.1 the pivot $B_{j} \rightarrow B_{j}^{\prime}$ strictly increases if and only if

$$
\chi_{\mathscr{D}}\left(\lambda_{j}, \lambda_{j}^{\prime}, i, i^{\prime}\right) \cdot \chi_{\mathscr{D}}\left(\lambda_{j}, \lambda_{j}^{\prime}, i, \lambda_{j-1}\right) \cdot \chi_{\mathscr{D}}\left(\lambda_{j}, \lambda_{j}^{\prime}, i, \lambda_{j+1}\right) \cdot \chi_{\mathscr{D}}\left(\lambda_{j}, \lambda_{j}^{\prime}, \lambda_{j-1}, \lambda_{j+1}\right)=+1
$$

Applying the definition of the lifting oriented matroid $\chi_{\mathscr{D}}$ we obtain the equivalent condition:

$$
-\Lambda\left(\lambda_{j}, i\right) \cdot \chi\left(\lambda_{j}, i, \lambda_{j-1}\right) \cdot \chi\left(\lambda_{j}, i, \lambda_{j+1}\right) \cdot \chi\left(\lambda_{j}, \lambda_{j-1}, \lambda_{j+1}\right)=+1
$$

Similarly the fact that $B_{j}^{\prime} \rightarrow B_{j+1}$ strictly increases translates to

$$
\chi_{\mathscr{D}}\left(\lambda_{j}, \lambda_{j+1}, i, i^{\prime}\right) \cdot \chi_{\mathscr{D}}\left(\lambda_{j}, \lambda_{j+1}, i, \lambda_{j}^{\prime}\right) \cdot \chi_{\mathscr{D}}\left(\lambda_{j}, \lambda_{j+1}, i, \lambda_{j+1}^{\prime}\right) \cdot \chi_{\mathscr{D}}\left(\lambda_{j}, \lambda_{j+1}, \lambda_{j}^{\prime}, \lambda_{j+1}^{\prime}\right)=+1,
$$

and, via the definition of $\chi_{\mathscr{D}}$ to

$$
\chi\left(\lambda_{j}, \lambda_{j+1}, i\right) \cdot \Lambda\left(\lambda_{j}, \lambda_{j+1}\right)=+1
$$

Conditions $(\mathrm{e} 1)_{j}$ and $(\mathrm{e} 2)_{j}$ show that negating the function $\Lambda$ changes the direction of all pivot operations, as claimed before. Simple multiplication of the terms now proves for $1 \leq j \leq k$ the following implications:

$$
\left.(\mathrm{e} 1)_{j} \text { and (ii) }\right)_{j} \text { implies }(\mathrm{e} 2)_{j}
$$

and

$$
\text { (e2) } \left.)_{j} \text { and (iii) }\right)_{j+1} \text { implies }(\mathrm{e} 1)_{j+1} \text {. }
$$

Since we assumed (e1), to be true, successive application of the above implications prove $(\mathrm{e} 1)_{j}$ and $(\mathrm{e} 2)_{j}$ for all $1 \leq j \leq k$. This proves the claim. 
As a direct consequence of Theorem 3.6 and Corollary 3.3 we now obtain:

Corollary 3.7. Noneuclidean link diagrams are not liftable.

Proof. If $\mathscr{D}$ is noneuclidean, then there exists an $i \in E$ such that $\left(\chi_{\mathscr{D}}, i, i^{\prime}\right)$ is noneuclidean. Therefore $\chi_{\mathscr{D}}$ is not realizable and $\mathscr{D}$ is not liftable.

Clearly, there are easier ways of proving the nonliftability of noneuclidean link diagram than proving noneuclideanness of $\chi_{\mathscr{D}}$. Nonetheless, noneuclidean oriented matroids are often considered as complicated geometric objects and it is usually difficult to understand their geometric content. Using the above concepts we have an easy tool to generate (and to visualize) noneuclidean rank 4 oriented matroids by describing a link diagram.

In [14] Mandel constructed a class $P_{n}^{d}$ of minor-minimal noneuclidean oriented matroids on $n$ elements in rank $d$ for every $d \geq 5$ and $n \geq 2 d$. Here "minorminimal" means that every proper minor of $P_{n}^{d}$ is euclidean. It remained open whether such a class existed for rank $d=4$ also. For the case of (ordinary) matroids (where it is also possible to define euclideanness) Bachem and Wanka [1], [2] proved that there exist minor-minimal noneuclidean classes for $d \geq 4$. However, in rank 4 every noneuclidean matroid contains an 8-element noneuclidean minor. We now construct a class of minor-minimal noneuclidean rank 4 oriented matroids. This closes the gap for the oriented matroid case and contrasts the matroid case.

First we define for all $n>3$ a link diagram $\mathscr{D}_{n}=\left(\chi_{n}, \Lambda_{n}\right)$ on $n$ elements $E_{n}:=\{1, \ldots, n\}$ which is the starting point of our construction. Here $\chi_{n}$ is the alternating oriented matroid defined by

$$
\chi_{n}(i, j, k)=+1 \quad \text { for } \quad 1 \leq i<j<k \leq n
$$

and $\Lambda_{n}$ is defined by

$$
\Lambda_{n}(i, j):= \begin{cases}0 & \text { if } i=j \\ +1 & \text { if } i=j+1 \text { or } i=j-1 \\ -1 & \text { otherwise, }\end{cases}
$$

indices counted modulo $n$.

Figure 6 represents a picture of $\mathscr{D}_{7}$. A special line arrangement realizing $\chi_{n}$ in the plane is given by the lines corresponding to the sides of a convex $n$-gon in the plane. We assume that for each $n>4$ such a special realization of $\chi$ is given by $\mathscr{L}_{n}$. Furthermore, we assume that in $\mathscr{L}_{n}$ no two lines are parallel and that the origin is chosen not collinear to each pair of intersections in $\mathscr{L}_{n}$ (this allows us to apply Theorem 2.2 later). The weaving diagram encoding $\mathscr{D}_{n}$ on the lines $\mathscr{L}_{n}$ is called $\mathscr{W}_{n}$. We first prove: 


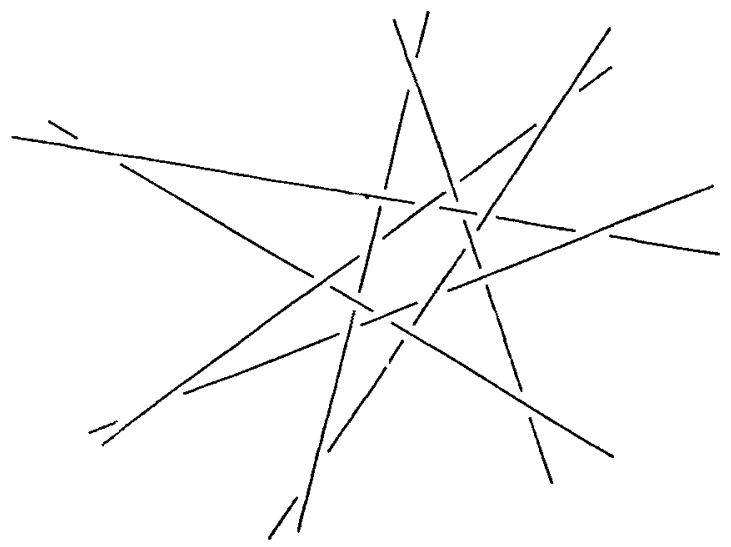

Fig. 6. The noneuclidean link diagram $\mathscr{D}_{7}$.

Lemma 3.8. The link program $\left(\mathscr{D}_{n}, i\right)$ is noneuclidean for every $i \in E_{n}$.

Proof. Since $\mathscr{D}$ is completely symmetric in all elements it is sufficient to prove this fact for the link program $(\mathscr{D}, 1)$. We prove that the sequence $2,3, \ldots, n$ is a nondegenerate cycle in $(\mathscr{D}, 1)$ by applying the characterization of lemma 3.5 . We get

$$
\begin{aligned}
& \chi_{n}(2,1,3) \cdot \chi_{n}(2,1, n) \cdot \Lambda_{n}(2,3) \cdot \Lambda_{n}(2, n)=-1, \\
& \chi_{n}(2,3, n) \cdot \chi_{n}(2,3,1) \cdot \Lambda_{n}(2, n) \cdot \Lambda_{n}(2,1)=-1, \\
& \chi_{n}(2, n, 1) \cdot \chi_{n}(2, n, 3) \cdot \Lambda_{n}(2,1) \cdot \Lambda_{n}(2,3)=-1,
\end{aligned}
$$

which proves that the above-below relations of line 2 with the lines 1,3 , and $n$ form an alternating sequence. Similarly, we prove that 1,2 , and $n-1$ alternate along $n$. We get

$$
\begin{aligned}
& \chi_{n}(n, 1,2) \cdot \chi_{n}(n, 1, n-1) \cdot \Lambda_{n}(n, 2) \cdot \Lambda_{n}(n, n-1)=-1, \\
& \chi_{n}(n, 2, n-1) \cdot \chi_{n}(n, 2,1) \cdot \Lambda_{n}(n, n-1) \cdot \Lambda_{n}(n, 1)=-1, \\
& \chi_{n}(n, n-1,1) \cdot \chi_{n}(n, n-1,2) \cdot \Lambda_{n}(n, 1) \cdot \Lambda_{n}(n, 2)=-1
\end{aligned}
$$

It remains to prove that $1, k-1$, and $k+1$ alternate along $k$ which is done by the following three equations:

$$
\begin{aligned}
& \chi_{n}(k, 1, k-1) \cdot \chi_{n}(k, 1, k+1) \cdot \Lambda_{n}(k, k-1) \cdot \Lambda_{n}(k, k+1)=-1, \\
& \chi_{n}(k, k-1,1) \cdot \chi_{n}(k, k-1, k+1) \cdot \Lambda_{n}(k, 1) \cdot \Lambda_{n}(k, k+1)=-1, \\
& \chi_{n}(k, k+1,1) \cdot \chi_{n}(k, k+1, k-1) \cdot \Lambda_{n}(k, 1) \cdot \Lambda_{n}(k, k-1)=-1 .
\end{aligned}
$$

We obtain that $(\mathscr{D}, i)$ is noneuclidean for every $i \in E_{n}$. 
Lemma 3.9. For each $i \in E_{n}$ the diagram $\mathscr{D}_{n} \backslash\{i\}$ (obtained by removing $l_{i}$ from $\mathscr{D}_{n}$ ) has a lifting $\mathscr{L}_{i, n}^{\prime}$ projecting on the lines $\mathscr{L}_{n} \backslash l_{i}$.

Proof. Again by the symmetry of $\mathscr{D}_{n}$ it is sufficient to prove the fact for $i=n$. We can prove the existence of the lifting by induction. Assume that for $k<n$ a lifting $\mathscr{L}_{k-1}^{\prime}$ of $\mathscr{D}_{n} \backslash\{k, \ldots, n\}$ is given that projects on the lines $\mathscr{L}_{n} \backslash\left(l_{k} \cup \cdots \cup l_{n}\right)$. In the weaving diagram $\mathscr{W}_{n} \backslash\{k+1, \ldots, n\}$ the above-below relations along line $k$ are split. This means that there exists a point $p$ on $l_{k}$ which subdivides $l_{k}$ in two half-lines such that all "above crossings" lie on one side of $p$ and all "below crossings" lie on the other side of $p$. In such a case we can enlarge our lifting $\mathscr{L}_{k-1}^{\prime}$ by a line $l_{k}^{\prime}$ in such a way that $\mathscr{L}_{k-1}^{\prime} \cup l_{k}^{\prime}$ forms a lifting of $\mathscr{D}_{n} \backslash\{k+1, \ldots, n\}$ and $l_{k}^{\prime}$ projects to $l_{k}$. The line $l_{k}^{\prime}$ can be constructed by first taking a line through the splitting point $p$ and parallel to the $z$-axis and then rotating it slightly in the plane $\pi^{-1}\left(l_{k}\right)$. Proceeding inductively proves the lemma.

A statement of how this splitting principle applies in general can be found in [17].

We are now ready to prove our main theorem:

Theorem 3.10. For every $n \geq 4$ the oriented matroid $M_{2 n}:=\chi\left[\mathscr{L}_{n}, o, \mathscr{D}_{n}\right]$ on $2 n$ elements is noneuclidean and every proper minor of $M_{2 n}$ is euclidean.

Proof. The oriented matroid $M_{2 n}$ is a specialization of the lifting oriented matroid $\chi_{\mathscr{D}_{n}}$. Since $\mathscr{D}_{n}$ was noneuclidean by Lemma 3.8 we can conclude by Theorem 3.6 that $M_{2 n}$ is also noneuclidean.

It remains to prove the minor-minimality of $M_{2 n}$. Every contraction of $M_{2 n}$ is of rank 3 and therefore euclidean (see Proposition 10.5.7 of [3]). It remains to show that after deletion of any element in $M_{2 n}$ we get a euclidean oriented matroid. Moreover, we prove that these deletions are indeed realizable. The symmetry of $\mathscr{D}_{n}$ allows us to consider only the deletions $M^{1}:=M_{2 n} \backslash\{n\}$ and $M^{2}:=M_{2 n} \backslash\left\{n^{\prime}\right\}$.

We modify our link diagram $\mathscr{D}_{n}$ into a link diagram $\mathscr{D}_{n}^{\prime}=\left(\chi_{n}, \Lambda_{n}^{\prime}\right)$ with

$$
\Lambda_{n}^{\prime}(i, j)= \begin{cases}-\Lambda(i, j) & \text { if }\{i, j\}=\{n, 1\} \quad \text { or } \quad\{i, j\}=\{n, n-1\} \\ \Lambda(i, j) & \text { otherwise. }\end{cases}
$$

Observe that $\mathscr{D}_{n}^{\prime}=\mathscr{D}_{n+1} \backslash\{n+1\}$.

By Lemma 3.9 there exists a lifting of $\mathscr{D}_{n}^{\prime}$ that projects on the lines $\mathscr{L}_{n}$. Therefore $\chi\left[\mathscr{L}_{n}, o, \mathscr{D}_{n}^{\prime}\right]$ is realizable by a suitable matrix $Y_{\varepsilon}$ via Theorem $2.2 . \chi\left[\mathscr{L}_{n}, o, \mathscr{D}_{n}^{\prime}\right]$ differs from $\mathscr{M}_{2 n}$ only in the signs $\left(1,1^{\prime}, n, n^{\prime}\right)$ and $\left(n-1,(n-1)^{\prime}, n, n^{\prime}\right)$. Therefore, $M^{1}$ and $M^{2}$ can be realized be deleting the last or penultimate row of $Y_{\varepsilon}$, respectively. 


\section{The Nonliftability of the Alternating Bipartite $4 \times 4$ Weaving Pattern}

In this section we want to investigate another setting where oriented matroids can be applied to solve liftability problems of link diagrams. Up to now a line in space was always considered to be the intersection of two planes. This approach was justified since for a given link diagram $\mathscr{D}=(\chi, \Lambda)$ we could arrange the planes in such a way that they form two bundles where one bundle completely describes the situation of the underlying planar projection of the lines.

Nonetheless, there are also situations where it can be useful to consider the lines in space as spanned by two points. When the link diagram contains many incidences between the lines this often leads to an easier description of the liftability condition. In this case a point in the intersection of two lines can be used in the description of both lines involved. Moreover, the occurrence of explicit incidences often simplifes the underlying algebraic structure of the liftability problem.

We exemplify this kind of thinking by giving a nonliftability proof for the alternating bipartite $4 \times 4$ weaving diagram. A geometric proof for the nonliftability of this diagram was also given by Pach et al. [16]. There the proof was given on four pages including the distinction of four different geometric situations. Here we give an algebraic proof that concludes the nonliftability as a consequence of a certain determinant identity. In principle, the proof can be done in a completely coordinate-free way involving only invariant theoretic-like calculations on determinants. However, to shorten the proof we introduce a specific coordinate system that allows us to make direct use of the occurring identities.

We want to prove that the bipartite alternating $4 \times 4$ weaving diagram $\mathscr{L}_{4,4}$ as shown in Fig. 7(a) is not liftable. more precisely:

Theorem 4.1 [16]. There is no liftable weaving diagram containing a substructure combinatorially equivalent to $\mathscr{L}_{4,4}$.

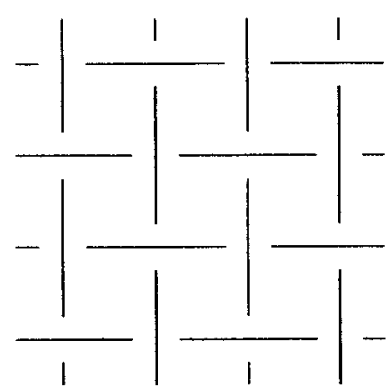

(a)

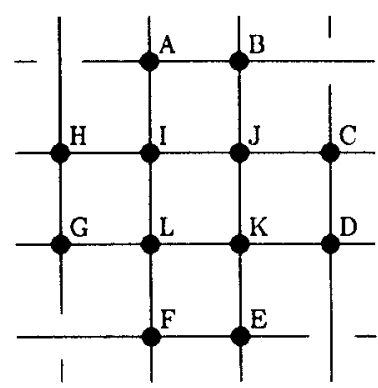

(b)

Fig. 7. The alternating $4 \times 4$ weaving diagram $\mathscr{L}_{4,4}$. 
Proof. In order to prove the nonliftability of $\mathscr{L}_{4,4}$ assume on the contrary that there are eight lines $\mathscr{L}:=\left\{l_{1}, \ldots, l_{8}\right\}$ in $\mathbb{R}^{3}$ projecting onto a partial diagram, which is combinatorially isomorphic to $\mathscr{L}_{4,4}$. After performing a suitable sequence of rotations of the lines in $\mathscr{L}$ it can be assumed that the lines intersect except for the four extreme vertices in the projection (see [16]). Therefore it remains to show that the partial diagram given in Fig. $7($ b) is not liftable.

Assume that there are 12 points $A, B, \ldots, L \in \mathbb{R}^{3}$ realizing the diagram in Fig. 7(b). We may consider them embedded into the three-dimensional real projective space and represented by homogeneous coordinates. Let $Y$ denote the corresponding $12 \times 4$ coordinate matrix and let $\chi:=\chi_{Y}$ be the underlying oriented matroid. We may furthermore assume that the four inner points $I, J, K$, and $L$ are not coplanar since in this case the whole configuration would be planar. Without loss of generality we may assume that the basis orientation of $(I, J, K, L)$ is positive (if not, a rotation of the whole configuration by $90^{\circ}$ and a relabeling of the points transforms the configuration to this case). For convenience identify the points $A, \ldots, L$ with their homogeneous coordinates and with their indices. We can read off the bases orientations of the points by the signs of the corresponding determinants in $Y$. Notice that, since the points $H, I, J$, and $C$ lie on one of the lines in this order, we can conclude

$$
\chi(H, I, K, L)=\chi(H, J, K, L)=\chi(I, C, K, L)=\chi(J, C, K, L)=\chi(I, J, K, L)=+1
$$

A similar statement holds for the remaining three interior lines. We get

$$
\begin{gathered}
\chi(B, J, L, I)=\chi(B, K, L, I)=\chi(K, E, L, I)=\chi(J, E, L, I)=\chi(J, K, L, I)=-1 \\
\chi(D, K, I, J)=\chi\left(D^{\prime}, L, I, J\right)=\chi(L, G, I, J)=\chi(K, G, I, J)=\chi(K, L, I, J)=+1 \\
\chi(F, L, J, K)=\chi(F, I, J, K)=\chi(I, A, J, K)=\chi(L, A, J, K)=\chi(L, I, J, K)=-1
\end{gathered}
$$

After a suitable projective transformation (with positive determinant) is performed the points $I, J, K$, and $L$ may be considered situated at the vertices of a regular tetrahedron given by the following projective coordinates:

$$
\left(\begin{array}{l}
I \\
J \\
K \\
L
\end{array}\right):=\left(\begin{array}{llll}
1 & 0 & 0 & 0 \\
0 & 1 & 0 & 0 \\
0 & 0 & 1 & 0 \\
0 & 0 & 0 & 1
\end{array}\right)
$$

Using the fact that the points $A, B, \ldots, H$ lie on suitable lines spanned by $I, J$, 
$K$, and $L$ we get the following representation of the configuration:

$$
\left(\begin{array}{l}
A \\
B \\
C \\
D \\
E \\
F \\
G \\
H \\
I \\
J \\
K \\
L
\end{array}\right):\left(\begin{array}{cccc}
a^{\prime} & 0 & 0 & a \\
0 & b^{\prime} & b & 0 \\
c & c^{\prime} & 0 & 0 \\
0 & 0 & d^{\prime} & d \\
0 & e & e^{\prime} & 0 \\
f & 0 & 0 & f^{\prime} \\
0 & 0 & g^{\prime} & g \\
h^{\prime} & h & 0 & 0 \\
1 & 0 & 0 & 0 \\
0 & 1 & 0 & 0 \\
0 & 0 & 1 & 0 \\
0 & 0 & 0 & 1
\end{array}\right)
$$

Our above sign conditions translate to the fact that the variables $a, \ldots, h$ and $a^{\prime}, \ldots, h^{\prime}$ are all negative. Furthermore, the four remaining above-below relations imply that the following four determinant conditions are satisfied:

$$
\begin{array}{ll}
\operatorname{det}(A, B, C, D)>0, & \operatorname{det}(C, D, E, F)>0, \\
\operatorname{det}(E, F, G, H)>0, & \operatorname{det}(G, H, A, B)>0 .
\end{array}
$$

An easy calculation shows

$$
\begin{aligned}
& \operatorname{det}(A, B, C, D)=a c b^{\prime} d^{\prime}-b d a^{\prime} c^{\prime}, \\
& \operatorname{det}(C, D, E, F)=d f c^{\prime} e^{\prime}-c e d^{\prime} f, \\
& \operatorname{det}(E, F, G, H)=e g f^{\prime} h^{\prime}-f h e^{\prime} g^{\prime}, \\
& \operatorname{det}(G, H, A, B)=b h a^{\prime} g^{\prime}-a g b^{\prime} h^{\prime} .
\end{aligned}
$$

Now the determinant inequalities (*) may be written as follows:

$$
\begin{gathered}
a c b^{\prime} d^{\prime}<b d a^{\prime} c^{\prime}, \\
d f c^{\prime} e^{\prime}<c e d^{\prime} f^{\prime}, \\
e g f^{\prime} h^{\prime}<f h e^{\prime} g^{\prime}, \\
b h a^{\prime} g^{\prime}<a g b^{\prime} h^{\prime} .
\end{gathered}
$$

Notice that all left and right sides of the inequalities are positive. Since every variable that appears on the left also appears on the right, multiplying all left and all right sides yields a contradiction. 


\section{References}

1. A. Bachem and A. Wanka: Separation theorems for oriented matroids. Discrete Math. 70 (1988), 303-310.

2. A. Bachem and A. Wanka: Euclidean intersection properties. J. Combin. Theory Ser. B 47 (1989), 10-19.

3. A. Björner, M. Las Vergnas, B. Sturmfels, N. White, and G. M. Ziegler: Oriented Matroids. Encyclopedia of Mathematics and Its Applications, Vol. 46. Cambridge University Press, Cambridge, 1993.

4. R. G. Bland: A combinatorial abstraction of linear programming. J. Combin. Theory Ser. B 23 (1977), 33-57.

5. J. Bokowski and J. Richter-Gebert: On the classification of non-realizable oriented matroids, Part II: properties. Preprint, TH-Darmstadt, 1990.

6. J. Bokowski and J. Richter: On the finding of final polynomials. European J. Combin. 11 (1990), 21-34.

7. J. Bokowski, J. Richter, and B. Sturmfels: Nonrealizability proofs in computational geometry. Discrete Comput. Geom. 5 (1990), 333-350.

8. J. Bokowski and B. Sturmfels: Computational Synthetic Geometry. Lecture Notes in Mathematics, Vol. 1355. Springer-Verlag, Berlin, 1989.

9. H. Crapo and R. Penne: Chirality and Isotopy of Lines in 3-Space. In preparation.

10. H. Crapo and J. Ryan: Spatial realizations of linear scenes. Structural Topology 13 (1986), 33-68.

11. J. Folkman and J. Lawrence: Oriented matroids. J. Combin. Theory Ser. B 25 (1978), 199-238.

12. K. Fukuda: Oriented Matroid Programming. Ph.D. Thesis, University of Waterloo, 1982.

13. H. Imai: On combinatorial structures of line drawings of polyhedra. Discrete Appl. Math. 10 (1985), 79-92.

14. A. Mandel: Topology of Oriented Matroids. Ph.D. Thesis, University of Waterloo, 1982.

15. V. F. Mazurovskii: Configurations of six skew lines. Zap. Nauchn. Sem. Leningrad. Otdel. Mat. Inst. Steklov. (L OMI) 167 (1988), 121-134.

16. J. Pach, R. Pollack, and E. Welzl: Weaving patterns of lines and line segments in space. Draft Preprint, Courant Institute, 1991.

17. R. Penne: Lines in 3-Space. Isotopy, Chirality and Weavings. Ph.D. Thesis, University of Antwerp (UIA), 1992.

18. R. Penne: Configurations of few lines in 3-Space. Isotopy, chirality, and planar layouts. Geom. Dedicata 45 (1993), 49-82.

19. J. Richter-Gebert: On the Realizability Problem of Combinatorial Geometries-Decision Methods. Dissertation, Technische Hochschule, Darmstadt, 1992.

20. J. Richter-Gebert: New Construction Methods for Oriented Matroids. Dissertation, KTH-Stockholm, 1992.

21. J. Richter-Gebert: Euclideanness and final polynomials in oriented matroid theory. Combinatorica, to appear.

22. J. Stolfi: Oriented projective geometry. Proceedings of the ACM Conference on Computational Geometry, Association for Computing Machinery, New York, 1987.

23. J. Stolf: Primitives for Computational Geometry. Report 36, Digital Systems Research Institute, Palo Alto, 1989.

24. K. Sugihara: A unifying approach to descriptive geometry and mechanisms. Discrete Appl. Math. 5 (1983), 313-328.

25. O. Ya. Viro: Topological problems concerning lines and points of three-dimensional space. Soviet Math. Dokl. 32 (1985), 528-531.

26. O. Ya. Viro and Yu. V. Drobotukhina: Configurations of skew lines. Leningrad Math. J. 1 (1990), $1027-1050$.

27. W. Whiteley: Rigidity and polarity II: weaving lines and tensegrity frameworks. Geom. Dedicata 30 (1989), 233-279.

Received April 17, 1992, and in revised form February 18, 1993. 